Check for updates

Cite this: RSC Adv., 2017, 7, 55812

\title{
Slippery surface based on lubricant infused hierarchical silicon nanowire film $\dagger$
}

\author{
Tian Hang, $\dot{t}^{\mathrm{a}}$ Hui-Jiuan Chen, $\dot{+}^{\mathrm{a}}$ Chengduan Yang, ${ }^{a}$ Shuai Xiao, ${ }^{a}$ Guishi Liu, ${ }^{\text {ab }}$ \\ Di-an Lin, ${ }^{a}$ Jun Tao, ${ }^{a}$ Jiangming Wu, ${ }^{a}$ Bo-ru Yang ${ }^{a}$ and Xi Xie ${ }^{1 D}$ *a
}

Nanowire thin films exhibiting superhydrophobicity or even superomniphobicity can protect themselves from liquid contamination. However, high contact angle of these thin films with liquid droplets limits the interfacial area between liquids and nanowire network, which is unfavorable for applications such as sensing suspended liquid droplets. In this study, slippery surface based on lubricant infused hierarchical Si nanowire film was developed, which provided low contact angle with liquid droplet, while possessing liquid repellent properties upon slight tilting. Si nanowire films were fabricated through chemical vapor deposition and were further branched with $\mathrm{ZnO}$ nanospikes for enhanced immobilization of lubricant. These hierarchical nanowire films were functionalized to enhance their superhydrophobicity and were further infused with perfluorinated lubricant to produce slippery surface. These infused slippery surfaces exhibited low contact angles with blood and corn oil along with high repellency towards these liquids. This study offers a promising approach for creating nanowire films that avoid liquid contamination.

Received 20th September 2017 Accepted 27th November 2017

DOI: $10.1039 / c 7 r a 10460 j$

rsc.li/rsc-advances topographically roughened structure and low surface energy of the substrate. Alternatively, a novel approach called slippery liquid infused porous surfaces (SLIPs) has been developed recently by infiltrating non-volatile lubricants into porous solids. ${ }^{15}$ The addition of a lubricant layer to porous surfaces generated structures with excellent liquid repellent properties towards a broad range of liquids. ${ }^{15-18}$

Moreover, semiconductor nanowire network thin films have attracted immense industrial and research interest owing to their potential applications in the fields of optics, electronics, energy conversion, and sensors. ${ }^{19-23}$ For example, one dimensional (1D) silicon nanowires with high surface-to-volume ratio have emerged as potential candidates for biochemical sensors, detection of electrical, mechanical or chemical signals in cells, detection of biomolecules, such as DNA, RNA, proteins, peptides, and small molecules including ions and organic compounds, and detection of gases, such as $\mathrm{CO}, \mathrm{H}_{2}$, and $\mathrm{O}_{2} \cdot{ }^{24-26}$ Over the past years, semiconductor nanowire networks have been synthesized for a wide range of materials, such as Si and group III-V compounds using both top-down and bottom-up approaches. ${ }^{27,28}$ With advanced nanofabrication techniques, morphology of the nanowire networks can be precisely controlled and consequently their mechanical, electronic, and optical properties can be fine-tuned. ${ }^{20,29-31}$ In addition to traditional 1D nanowires, recent progress in fabrication of hierarchical branched structures has further stimulated related research. The existence of secondary nanowire branches increases the effective surface area, displaying improved properties and sensitivities of branched structures compared to 1D nanowire structures. ${ }^{32}$ Since micro-nano hierarchical structure

\footnotetext{
State Key Laboratory of Optoelectronic Materials and Technologies, School of Electronics and Information Technology, The First Affiliated Hospital of Sun Yat-Sen University, Guangdong Province Key Laboratory of Display Material and Technology, Sun Yat-Sen University, Guangzhou, China.E-mail: xiexi27@mail.sysu.edu.cn

${ }^{b}$ Wellman Center for Photomedicine, Massachusetts General Hospital, Department of Dermatology, Harvard Medical School, MA, USA

$\dagger$ Electronic supplementary information (ESI) available. See DOI: 10.1039/c7ra10460j

\$ These authors contributed equally to this work.
} 
(a)
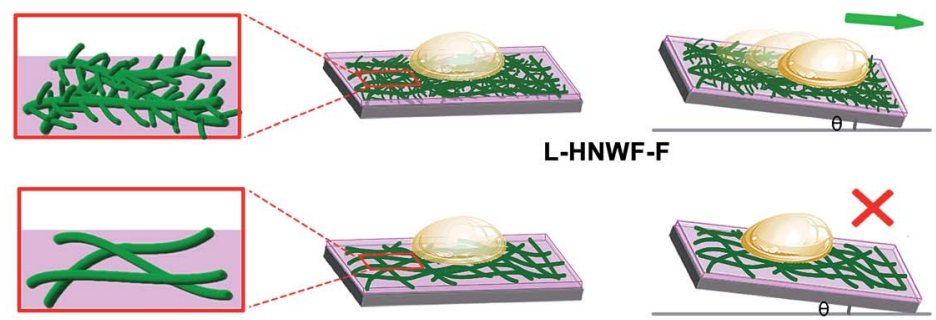

L-NWF-F

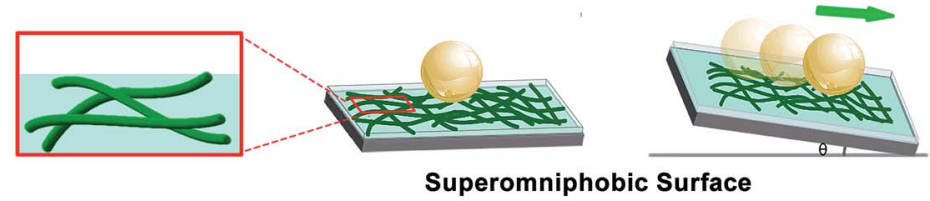

Superomniphobic Surface

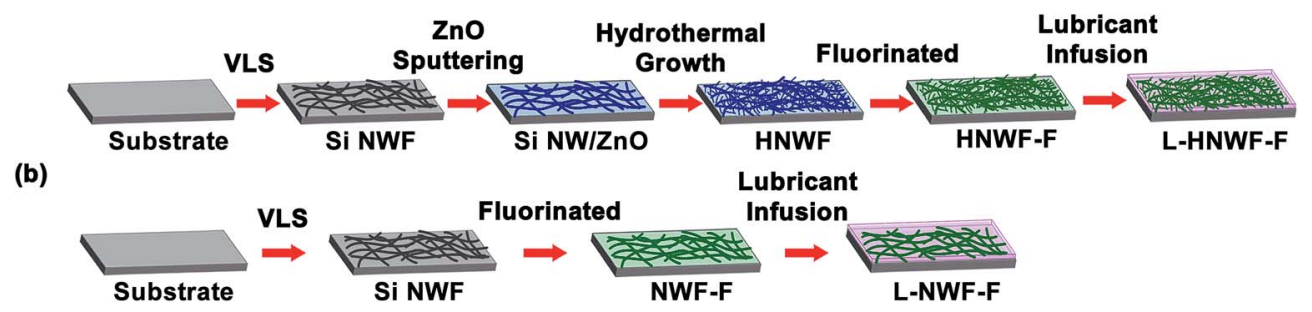

Fig. 1 (a) Illustration of the liquid contact and liquid repelling on lubricant infused hierarchical nanowire film, lubricant infused nanowire film, and conventional superomniphobic surface. (b) Schematic of the fabrication of lubricant infused hierarchical nanowire film and lubricant infused nanowire film.

is crucial to wetting property, hierarchical nanowires with branched structures offer a reasonable design for constructing hydrophobic or omniphobic surfaces. ${ }^{13,33}$

Despite its promising applications, nanowire thin films as sensors or probes tend to be contaminated by water, dust, oil, or dirt in liquids, which limit their lifetime, stability and durability. ${ }^{34}$ Superhydrophobic or even omniphobic nanowire thin films can provide ultra-high liquid contact angle on the film surface, thus allowing the surface to attain self-cleaning capability and protection from liquid contaminations. However, for sensing or detection of targets inside suspended liquid droplets, superhydrophobic or omniphobic nanowire thin films possesses high contact angle with the liquid droplet, thus limiting contact area between the liquids and the nanowire network and restricting their sensing capability. This is unfavorable for applications where liquid droplets need to sufficiently contact with or wet the solid surface.

In this study, slippery surfaces based on lubricating liquid infused hierarchical Si nanowire films were developed, which allowed liquid to contact with the surface at a smaller angle $\left(<100^{\circ}\right)$, while the nanowire films were capable of repelling various liquids upon slight tilting (Fig. 1a). The Si nanowire films were fabricated through chemical vapor deposition. To enhance lubricant locking within the nanowire film, the nanowires were branched with secondary $\mathrm{ZnO}$ nanowires through a hydrothermal approach. The hierarchical nanowire film (HNWF) was functionalized with fluorinated silane to produce low surface energy (HNWF-F), and it exhibited increased superhydrophobicity with excellent water-repelling properties compared to the fluorinated Si nanowire film without branched
ZnO nanowires (NWF-F). The hierarchical nanowire film was further infused with lubricant to produce slippery surface (LHNWF-F), which exhibited lower contact angle with blood and corn oil, while these liquids could readily slide-off upon slight tilting. In contrast, in case of liquids adhered to the lubricantinfused Si nanowire film (L-NWF-F), the hierarchical nanowires provided additional contact surface area with the lubricant and enhanced locking effects of lubricant. Our study illustrates the advantage of employing hierarchical nanowire design to enable excellent water or oil-repelling properties, thus providing a novel approach to synthesize nanowire films with liquid repellent property, which is important to protect nanowire sensors from contamination.

\section{Results}

The schematic of the fabrication of hierarchical nanowire films is illustrated in Fig. 1b. Semiconductor Si nanowire network films were synthesized through gold-catalytic vapour-liquidsolid approach..$^{35,36}$ Initially, $50 \mathrm{~nm}$ Au nanoparticles, which served as the catalyst for nucleation and growth of Si nanowires, were dispersed on $\mathrm{SiO}_{2} / \mathrm{Si}$ substrate. Si nanowires were grown at $450{ }^{\circ} \mathrm{C}$ for $1.5 \mathrm{~h}$ with $\mathrm{SiH}_{4}$ as the silicon reactant and $\mathrm{H}_{2}$ as the carrier gas. As shown in SEM images (Fig. 2), Si nanowires with an average diameter of $50 \mathrm{~nm}$ and length more than $10 \mu \mathrm{m}$ were produced uniformly. The nanowires were randomly oriented with high density of intermeshing, forming a continuous thin film network on the $\mathrm{SiO}_{2} / \mathrm{Si}$ substrate. To achieve hierarchical structure, branched $\mathrm{ZnO}$ nanowires were fabricated on the $\mathrm{Si}$ nanowire surface through a hydrothermal method. ${ }^{37}$ Initially, 

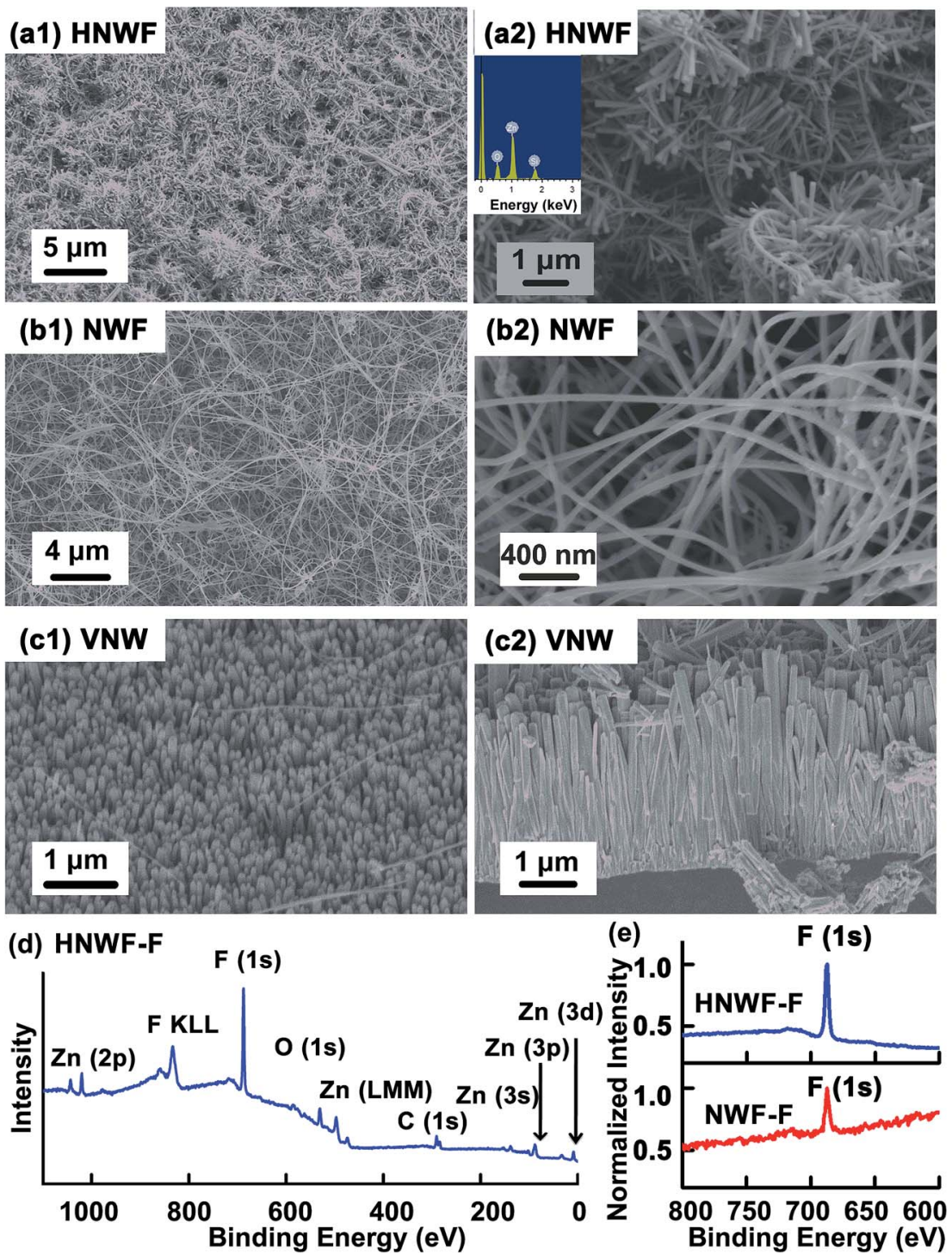

Fig. 2 SEM images of (a) hierarchical nanowire film, (b) 1D Si nanowire film, and (c) vertical ZnO nanowires. (1) and (2) are for low and high magnifications; the inset shows the EDS spectrum of hierarchical nanowire film. (d) XPS spectrum of fluorinated hierarchical nanowire film. (e) The F (1s) peak of the XPS spectrum indicated successful fluorination on both hierarchical nanowire film and 1D Si nanowire film.

$50 \mathrm{~nm} \mathrm{ZnO}$ thin layer was sputtered on the surface of Si nanowire films as a seed layer for $\mathrm{ZnO}$ nanowire growth. The entire substrate was immersed in an aqueous solution containing $25 \mathrm{mM}$ of zinc nitrate hydrate $\left[\mathrm{Zn}\left(\mathrm{NO}_{3}\right)_{2} \cdot 6 \mathrm{H}_{2} \mathrm{O}\right]$ and $25 \mathrm{mM}$ of hexamethylenetetramine $\left(\mathrm{C}_{6} \mathrm{H}_{12} \mathrm{~N}_{4}\right.$, HMTA $)$ and incubated at $80^{\circ} \mathrm{C}$ for $2 \mathrm{~h}$, which resulted in the growth of $\mathrm{ZnO}$ nanospikes.

As shown in Fig. 2a, the branched structure formed by the secondary $\mathrm{ZnO}$ nanospikes could be clearly observed on the backbones of Si nanowires. The $\mathrm{ZnO}$ nanowires are approximately $90 \mathrm{~nm}$ in diameter and $1 \mu \mathrm{m}$ in length. In addition, energy dispersion spectroscopy (EDS) was applied to evaluate the chemical composition of hierarchical nanowire films. The EDS spectrum verified that the branched structure was primarily composed of $\mathrm{ZnO}$. The high density of the branches on the surface of $\mathrm{Si}$ nanowires results in a denser network structure compared to the 1D Si nanowire network, creating a larger effective surface area that could be important for entrapping more air to suspend liquids according to Cassie model and for better immobilization of lubricant to produce a slippery surface. The hierarchical nanowire film was functionalized with perfluorooctyltriethoxysilane to provide low surface energy and chemical affinity that allows for the wetting and adhesion of perfluorinated lubricants on the nanowire surface. ${ }^{15,38} \mathrm{X}$-ray photoelectron spectroscopy was applied to characterize the surface chemical composition of the hierarchical nanowire films after surface functionalization. As shown in Fig. 2d, pronounced peak at $686 \mathrm{eV}$, corresponding to fluorine, evidenced that the hierarchical nanowires were successfully coated with fluorinated silane. Si nanowire films without ZnO nanowire branches were also fluorinated as control. Similarly, the XPS spectra of the control exhibited F peak, indicating successful conjugation of perfluorooctyltriethoxysilane on the Si nanowires (Fig. 2e). 
(a)
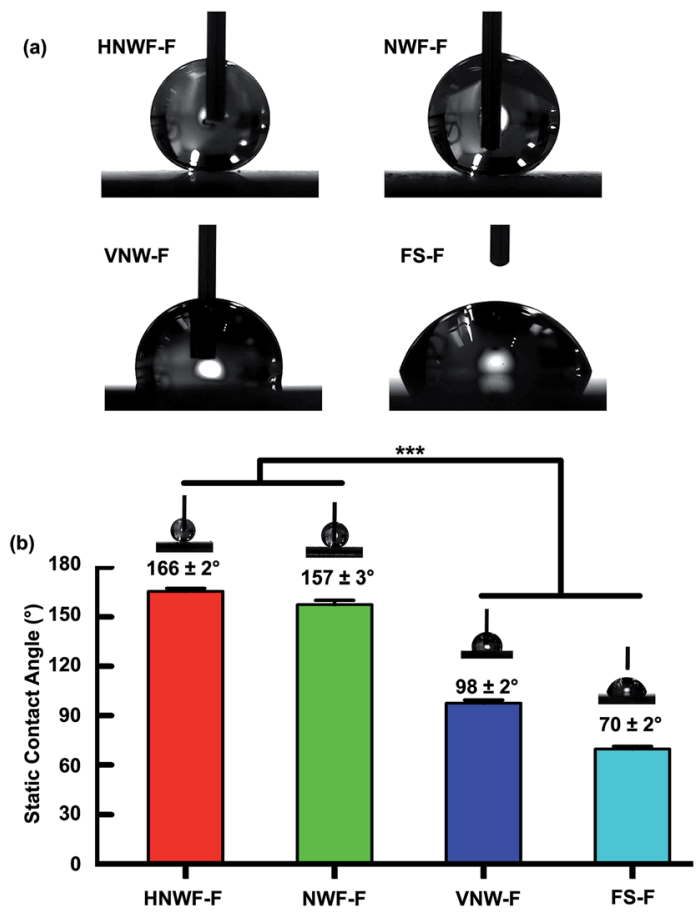

(c)

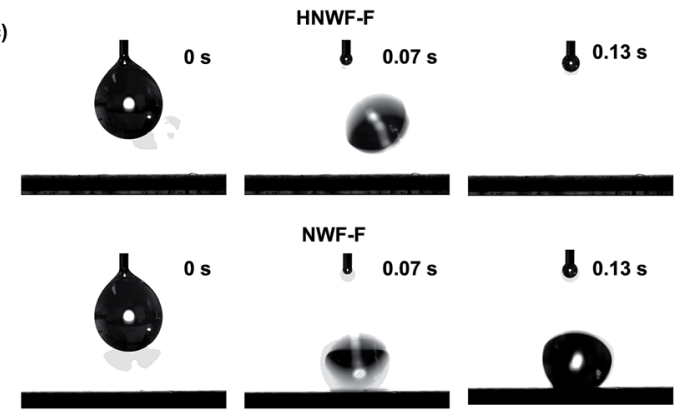

Fig. 3 (a) Optical image and (b) statistical results of the static contact angles of water on different surfaces including HNWF-F, NWF-F, VNW-F, and FS-F. $* * * p<0.001$ (c) time-resolved images of water drop $(5 \mu \mathrm{L})$ bouncing experiment, where the water droplet bounced off the HNWF-F surface while stuck to the NWF-F surface.

Successful fluorination would result in low surface energy, enabling superhydrophobicity of the surface. The wetting behavior of water on the fluorinated hierarchical nanowire film was studied by measuring the static contact angle (CA). In general, the CAs of water for both hierarchical and 1D Si nanowire film were found to increase after fluorination (Table S1 in ESI $†$ ). Fig. 3a and b show the CA of water drops on different surfaces: fluorinated hierarchical nanowire films (HNWF-F), and control samples including fluorinated Si NW films (NWF-F), fluorinated $\mathrm{ZnO}$ vertical nanowires (VNW-F), and fluorinated flat glass substrate (FS-F). The ${ }^{* * *}$ indicates significant differences $(p<0.001)$ of water CAs for HNWF-F and NWFF samples in comparison with VNW-F and FS-F samples. The CA of water on VNW-F was $98 \pm 2^{\circ}$, while both HNWF-F and NWF-F exhibited high contact angles (larger than $150^{\circ}$ ), which are significantly higher than on FS-F (contact angle was $70 \pm 2^{\circ}$ ). These results agree with previous studies, which revealed the (a)

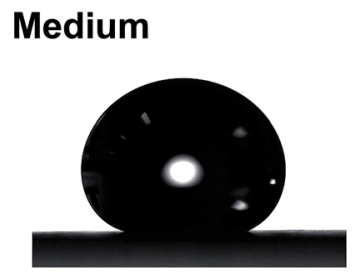

HNWF-F

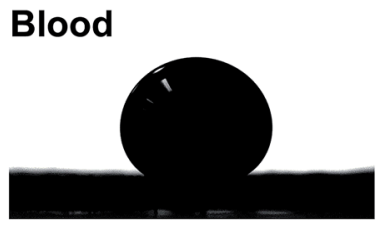

HNWF-F
Medium

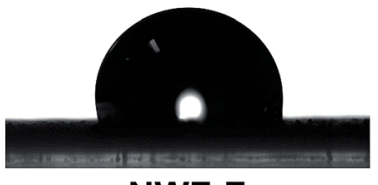

NWF-F

Blood

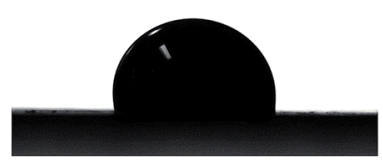

NWF-F (b)

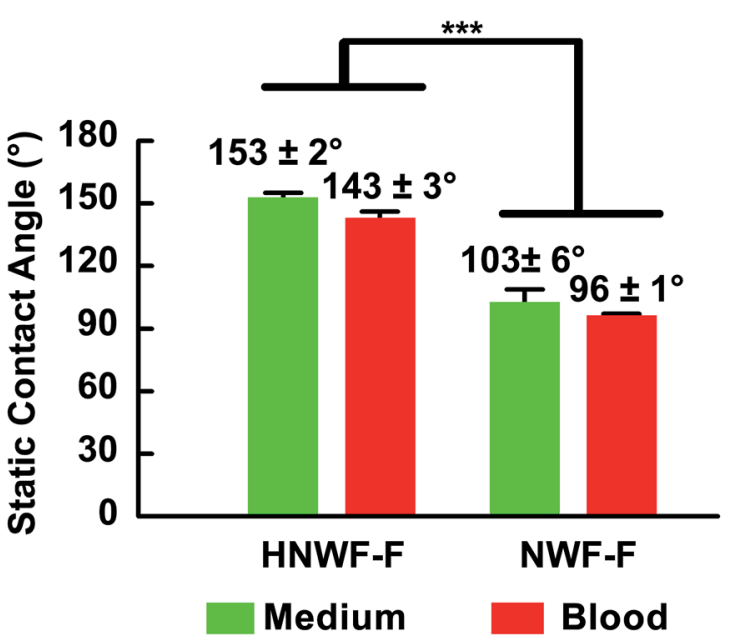

Fig. 4 (a) Optical image and (b) statistical results of the static contact angles of cell medium and blood on HNWF-F and NWF-F. ***p < 0.001 .

crucial role of microscale or nanoscale structures in enhancing non-wetting properties. ${ }^{39}$

In addition, a bouncing experiment was conducted by studying the sliding behavior of free-falling water droplets onto the object surfaces with $5^{\circ}$ tilting. As shown in Fig. $3 \mathrm{c}$, the water drop can easily bounce off the HNWF-F surface, indicating that the surface of hierarchical nanowires is highly repellent to water. In contrast, water drops stuck to the surface of NWF-F without rolling off. The introduction of $\mathrm{ZnO}$ nanowire branches on $\mathrm{Si}$ nanowire film resulted in a hierarchically textured structure, which entraps a higher fraction of air. As predicted by the Cassie model, these trapped air pockets could support the liquid droplet on top of the surface, contributing to a higher contact angle. Our obtained results were consistent with previous studies on designing superhydrophobic surfaces, which revealed that increased levels of hierarchy could enhance liquid-repelling behavior of the superhydrophobic surface. ${ }^{13}$

In addition to the wetting behavior of water on the fluorinated hierarchical nanowire films, the contact angle of complex fluids such as cell culture medium and blood (mice blood 
(a)

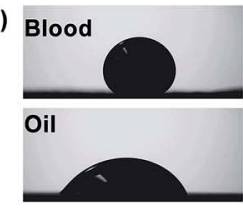

HNWF-F

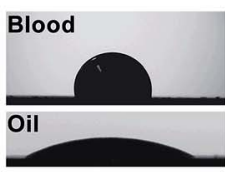

NWF-F

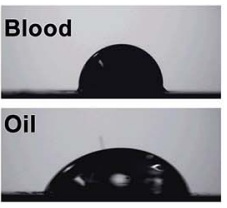

L-HNWF-F

(b)

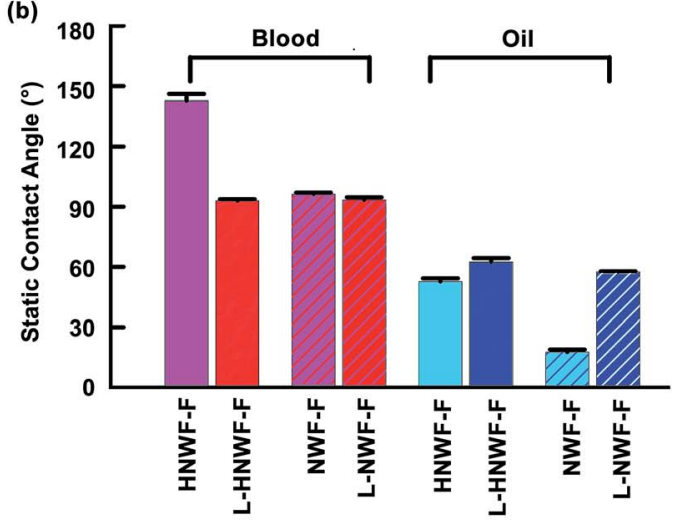

(c)

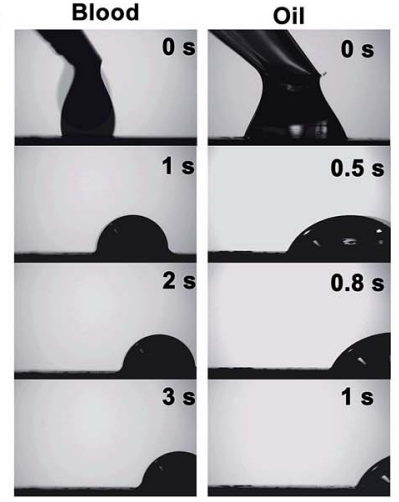

L-HNWF-F

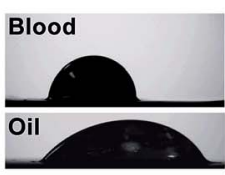

L-NWF-F

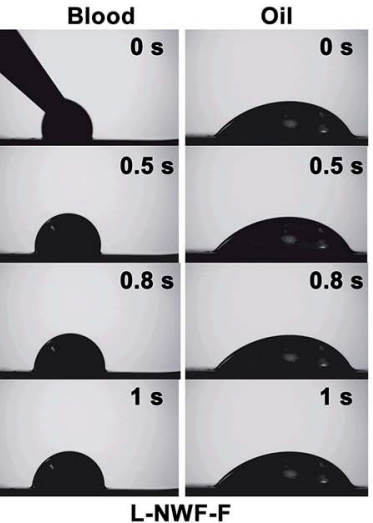

(d)

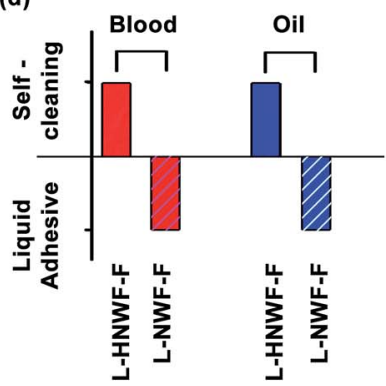

Fig. 5 (a) Optical image and (b) statistical results of the static contact angles of blood and oil on HNWF-F and NWF-F. (c) Sequential photographic images and (d) result summary showing the liquid mobility of blood and oil drops sliding on L-HNWF-F while adhered on L-NWF-F.

supplied by Laboratory Animal Center, Sun Yat-Sen University) were tested. Due to the presence of complex molecules such as amino acids, proteins and other organic compounds in cell culture medium and blood, these liquids tend to easily contaminate and stick on the surface of the nanowire films, presenting lower contact angle than pure water. Similar to the contact angle with water, the contact angles toward cell culture medium and blood were greatly increased after fluorination of the Si nanowires (Table S1 in ESI $\dagger$ ). The HNWF-F exhibited contact angles were $153 \pm 2^{\circ}$ to cell culture medium, and $143 \pm$ $3^{\circ}$ to blood, while NWF-F showed lower contact angle of $103 \pm$ $6^{\circ}$ to cell culture medium, and $96 \pm 1^{\circ}$ to blood (Fig. 4). A significant difference could be observed between HNWF-F and NWF-F $(p<0.001)$. These results further demonstrate that the secondary ZnO nanospikes in HNWF-F increased liquid contact angle on their surfaces, suggesting that a hierarchical structure is favorable for liquid-repelling properties. The enhancement of superhydrophobicity also indicated that the HNWF-F exhibited lower surface energy, which was favorable for lubricant immobilization.

Despite the presented superhydrophobicity of modified $\mathrm{Si}$ nanowires, repelling of low surface-tension liquids is challenging even with hierarchical structures (Fig. 5a). In addition, the design principle of superomniphobicity, involving the reduction of contact points with liquid droplets, limits liquid contact with the solid surface, which is unfavorable for applications, in which liquid droplets are required to have sufficient contact area with the solid surface. On this basis, the recently developed slippery liquid infused porous surfaces (SLIPs) technique can overcome this challenge. ${ }^{40}$ Lubricants can repel many types of low surface-tension liquids, such as oil or nonpolar solvents, by providing an immiscible fluid-fluid interface. For a stable slippery surface, the surface needs to possess sufficient chemical affinity with the lubricant. The introduction of $\mathrm{ZnO}$ branches produced a denser network structure with extra micro-nanoscale porosity compared to the 1D Si nanowire film without $\mathrm{ZnO}$ nanospikes, thus serving as a promising candidate substrate for fabricating SLIPs. Accordingly, to investigate the possibility of liquid repellency towards low surface tension liquids such as oil, the HNWF-F was infused with perfluorinated fluid to produce a slippery surface. This slippery surface was generated by placing a perfluorinated lubricant liquid, DuPont Krytox 100, on top of the substrate to allow the infusion of lubricant into the porous nanowire network, followed by removal of excess lubricant. ${ }^{18}$

Fig. 5 shows the wetting behavior of oil and blood droplets on the as-fabricated L-HNWF-F and L-NWF-F (as control). The LHNWF-F exhibited contact angles of $93 \pm 1^{\circ}$ to blood droplet and $63 \pm 2^{\circ}$ to oil droplets while L-NWF-F showed contact angle of $93 \pm 1^{\circ}$ to blood droplet and $58 \pm 1^{\circ}$ to oil droplet. The static 
contact angle is reduced on L-HNWF-F surface compared to that on HNWF-F, on which the blood and oil droplets spread flatter as shown in Fig. 5a. These results indicate that slippery surfaces allow immiscible liquids to exhibit lower contact angle, which are consistent with previous studies. ${ }^{2}$ Despite lower contact angle, oil and blood drops were highly mobile and could easily slide off the L-HNWF-F within a few seconds at a low tilt angle of $5^{\circ}$. In contrast, neither oil nor blood drops could slide off LNWF-F at the same tilt angle. This suggests that the hierarchical structure provided by the $\mathrm{ZnO}$ branches could stably entrap the lubricant fluid and hence improved the slippage of liquid drops. Our obtained results agree with previous studies about the effects of surface morphology on the performance of SLIPs. ${ }^{12,41-43}$ In general, surface roughness is essential to achieve stable slippery behavior. ${ }^{12}$ For example, Wang et al. observed that the substrate structure of SLIPs with a lower length scale (100-200 nm pore-like structure verses 1-2 $\mu \mathrm{m}$ pillar-like structure) provided better mobility of water drops. It was suggested that the high vacancy in microstructure could decrease the stability of lubricants. ${ }^{41}$ In our current study, the hierarchical nanowire film presented a denser network structure compared to the 1D nanowire film. The gaps between the $\mathrm{Si}$ nanowires may turn to the pinning sources, reducing lubricant locking, while the hierarchical nanowire network can provide better immobilization of lubricant, thus resulting in enhanced slippery performance.

\section{Conclusion}

In conclusion, hierarchical Si nanowire films were infused with a lubricant fluid to produce slippery surface that presented low contact angle with various liquids, including blood and corn oil, while the nanowire films were able to readily repel these liquids, thus protecting themselves from liquid contamination. The presence of $\mathrm{ZnO}$ branches increased the entrapped air fraction in the structure, thus providing improved superhydrophobic property. Moreover, the increased hierarchy of nanowire structure presented a denser network structure that provided extra contact surface with the lubricant, thus enhancing the immobilization of lubricant and presenting better liquid repellent properties compared to $1 \mathrm{D}$ nanowire films. Our study demonstrated a promising approach for the synthesis of hierarchical nanowires, which possess excellent liquid repellent properties and protect nanowire sensors from liquid contamination, thus improving sensor stability and durability.

\section{Conflicts of interest}

There are no conflicts to declare.

\section{Acknowledgements}

The authors wish to thank the Youth 1000 Talents Program of China and 100 Talents Program of Sun Yat-Sen University (76120-18821104) for supports. The authors would like to acknowledge financial support from the National Natural
Science Foundation of China (Grant No. 51705543, 61771498 and 31530023).

\section{References}

1 H. Wang, Y. Xue and T. Lin, Soft Matter, 2011, 7, 8158-8161.

2 C. Shillingford, N. MacCallum, T. S. Wong, P. Kim and J. Aizenberg, Nanotechnology, 2014, 25, 014019.

3 P. Guo, Y. Zheng, M. Wen, C. Song, Y. Lin and L. Jiang, $A d v$. Mater., 2012, 24, 2642-2648.

4 J. Lv, Y. Song, L. Jiang and J. Wang, ACS Nano, 2014, 8, 31523169.

5 G. D. Bixler and B. Bhushan, Soft Matter, 2013, 9, 1620-1635. 6 S. Li, J. Huang, M. Ge, C. Cao, S. Deng, S. Zhang, G. Chen, K. Zhang, S. S. Al-Deyab and Y. Lai, Adv. Mater. Interfaces, 2015, 2, 1600152.

7 R. Du, X. Gao, Q. Feng, Q. Zhao, P. Li, S. Deng, L. Shi and J. Zhang, Adv. Mater., 2016, 28, 936-942.

8 E. Jenner and B. D'Urso, Appl. Phys. Lett., 2013, 103, 251606.

9 E. Bormashenko, Adv. Colloid Interface Sci., 2015, 222, 92103.

10 C. Neinhuis and W. Barthlott, Ann. Bot., 1997, 79, 667-677.

11 A. K. Kota, G. Kwon and A. Tuteja, NPG Asia Mater., 2014, 6, e109.

12 P. Kim, M. J. Kreder, J. Alvarenga and J. Aizenberg, Nano Lett., 2013, 13, 1793-1799.

13 A. R. Bielinski, B. Boban, Y. He, E. Kazyak, D. H. Lee, C. Wang, A. Tuteja and N. P. Dasgupta, ACS Nano, 2017, 11, 478-489.

14 S. Zhang, J. Huang, Y. Tang, S. Li, M. Ge, Z. Chen, K. Zhang and Y. Lai, Small, 2016, 1-8.

15 T. S. Wong, S. H. Kang, S. K. Tang, E. J. Smythe, B. D. Hatton, A. Grinthal and J. Aizenberg, Nature, 2011, 477, 443-447.

16 N. MacCallum, C. Howell, P. Kim, D. Sun, R. Friedlander, J. Ranisau, O. Ahanotu, J. J. Lin, A. Vena, B. Hatton, T.-S. Wong and J. Aizenberg, ACS Biomater. Sci. Eng., 2015, 1, 43-51.

17 A. Grinthal and J. Aizenberg, Chem. Mater., 2014, 26, 698708.

18 A. K. Epstein, T.-S. Wong, R. A. Belisle, E. M. Boggs and J. Aizenberg, Proc. Natl. Acad. Sci. U. S. A., 2012, 109, 13182-13187.

19 W. Zhou, X. Dai and C. M. Lieber, Rep. Prog. Phys., 2017, 80, 016701.

20 X. Duan and C. M. Lieber, Nano Res., 2015, 8, 1-22.

21 X. Xie, A. Aalipour, S. V. Gupta and N. A. Melosh, ACS Nano, 2015, 9, 11667-11677.

22 X. Xie, A. M. Xu, M. R. Angle, N. Tayebi, P. Verma and N. A. Melosh, Nano Lett., 2013, 13, 6002-6008.

23 X. Xie, A. M. Xu, S. Leal-Ortiz, Y. H. Cao, C. C. Garner and N. A. Melosh, ACS Nano, 2013, 7, 4351-4358.

24 Y. Wang, T. Wang, P. Da, M. Xu, H. Wu and G. Zheng, Adv. Mater., 2013, 25, 5177-5195.

25 J. Zimmerman, R. Parameswaran and B. Tian, Biomater. Sci., 2014, 2, 619-626.

26 Z. Li, Y. Chen, X. Li, T. I. Kamins, K. Nauka and R. S. Williams, Nano Lett., 2004, 4, 245-247. 
27 T. Mikolajick, A. Heinzig, J. Trommer, S. Pregl, M. Grube, G. Cuniberti and W. M. Weber, Phys. Status Solidi RRL, 2013, 7, 793-799.

28 F. Ishikawa, in Quantum Sensing and Nano Electronics and Photonics Xiii, ed. M. Razeghi, G. J. Brown and J. S. Lewis, 2016, vol. 9755.

29 X. Jiang, B. Tian, J. Xiang, F. Qian, G. Zheng, H. Wang, L. Mai and C. M. Lieber, Proc. Natl. Acad. Sci. U. S. A., 2011, 108, 12212-12216.

30 M. Kwiat, S. Cohen, A. Pevzner and F. Patolsky, Nano Today, 2013, 8, 677-694.

31 E. Comini, Mater. Today, 2016, 19, 559-567.

32 X. Duan, R. Gao, P. Xie, T. Cohen-Karni, Q. Qing, H. S. Choe, B. Tian, X. Jiang and C. M. Lieber, Nat. Nanotechnol., 2012, 7, 174-179.

33 M. Zhang, P. Wang, H. Y. Sun and Z. K. Wang, ACS Appl. Mater. Interfaces, 2014, 6, 22108-22115.

34 J. Wang, Y. Zhang, S. Wang, Y. Song and L. Jiang, Acc. Chem. Res., 2011, 44, 405-415.
35 J. Yao, H. Yan and C. M. Lieber, Nat. Nanotechnol., 2013, 8, 329-335.

36 R. S. Wagner and W. C. Ellis, Appl. Phys. Lett., 1964, 4, 89-90. 37 S. H. Ko, D. Lee, H. W. Kang, K. H. Nam, J. Y. Yeo, S. J. Hong, C. P. Grigoropoulos and H. J. Sung, Nano Lett., 2011, 11, 666671.

38 P. Kim, T.-S. Wong, J. Alvarenga, M. J. Kreder, W. E. AdornoMartinez and J. Aizenberg, ACS Nano, 2012, 6, 6569-6577.

39 J. Shieh, F. J. Hou, Y. C. Chen, H. M. Chen, S. P. Yang, C. C. Cheng and H. L. Chen, Adv. Mater., 2010, 22, 597-601.

40 F. Schellenberger, J. Xie, N. Encinas, A. Hardy, M. Klapper, P. Papadopoulos, H.-J. Butt and D. Vollmer, Soft Matter, 2015, 11, 7617-7626.

41 P. Wang, D. Zhang and Z. Lu, Colloids Surf., B, 2015, 136, 240-247.

42 I. Okada and S. Shiratori, ACS Appl. Mater. Interfaces, 2014, 6, 1502-1508.

43 N. Vogel, R. A. Belisle, B. Hatton, T. S. Wong and J. Aizenberg, Nat. Commun., 2013, 4, 2176. 